\title{
SNAI transcription factors mediate epithelial- mesenchymal transition in lung fibrosis
}

\author{
A Jayachandran, ${ }^{1}$ M Königshoff,, ${ }^{2}$ Y Yu, ${ }^{1}$ E Rupniewska, ${ }^{1}$ M Hecker, ${ }^{1}$ W Klepetko, ${ }^{3}$ \\ W Seeger, ${ }^{1} 0$ Eickelberg ${ }^{2}$
}

- Additional tables, figures and experimental procedures are published online only at http:// thorax.bmj.com/content/vo64/ issue 12

${ }^{1}$ Department of Medicine, University of Giessen Lung Center, University of Giessen, Giessen, Germany;

${ }^{2}$ Comprehensive Pneumology Center, Institute of Lung Biology and Disease (iLBD), Ludwig-

Maximilians-University and Helmholtz Zentrum München, Neuherberg/Munich, Germany;

${ }^{3}$ Department of Thoracic

Surgery, University Hospital

Vienna, Vienna, Austria

Correspondence to: Professor Dr 0 Eickelberg, Comprehensive Pneumology Center, Institute of Lung Biology and Disease (iLBD). Helmholtz Zentrum München, Ingolstädter Landstraße 1, D-85764 Neuherberg/München, Germany; oliver.eickelberg@helmholtzmuenchen.de

Received 17 June 2009 Accepted 22 September 2009 Published Online First 22 October 2009

\section{ABSTRACT}

Background: Idiopathic pulmonary fibrosis (IPF) is a fatal interstitial lung disease characterised by accumulation of activated (myo)fibroblasts and excessive extracellular matrix deposition. The enhanced accumulation of (myo)fibroblasts may be attributed, in part, to the process of transforming growth factor $\beta 1$ (TGF $\beta 1$ )-induced epithelial-mesenchymal transition (EMT), the phenotypic switching of epithelial to fibroblast-like cells. Although alveolar epithelial type II (ATII) cells have been shown to undergo EMT, the precise mediators and mechanisms remain to be resolved. The objective of this study is to investigate the role of SNAI transcription factors in the process of EMT and in IPF.

Methods: Using quantitative reverse transcription-PCR (RT-PCR), immunofluorescence, immunohistochemistry, western blotting, as well as gain- and loss-of-function studies and functional assays, the role of SNAI1 and SNAI2 in TGF $\beta 1$-induced EMT in ATII cells in vitro was assessed; and the expression of SNAI transcription factors was analysed in experimental and human IPF in vivo.

Results: TGF $\beta 1$ treatment increased the expression and nuclear accumulation of SNAI1 and SNAI2, in concert with induction of EMT in ATII cells. SNAI overexpression was sufficient to induce EMT, and small interfering RNA (siRNA)-mediated SNAI depletion attenuated TGF $\beta 1$ induced ATIl cell migration and EMT. SNAI expression was elevated in experimental and human IPF and localised to hyperplastic ATII cells in vivo.

Conclusions: The results demonstrate that TGF $\beta 1$ induced EMT in ATII cells is essentially controlled by the expression and nuclear translocation of SNAI transcription factors. Increased SNAI1 and SNAI2 expression in experimental and human IPF in vivo suggests that SNAImediated EMT may contribute to the fibroblast pool in idiopathic pulmonary fibrosis.

Idiopathic pulmonary fibrosis (IPF) is a progressive and lethal disorder of major concern due to its unresolved pathogenesis and limited responsiveness to currently available therapies. ${ }^{1}$ The hallmark lesions of IPF are fibroblast foci, which are sites featuring $\alpha$-smooth muscle actin ( $\alpha \mathrm{SMA})$-positive, activated (myo)fibroblasts. ${ }^{2}$ Currently, three major theories attempt to explain the accumulation of activated (myo)fibroblasts in the lungs of patients with IPF. First, local fibroproliferation of resident pulmonary fibroblasts in response to fibrogenic cytokines and growth factors increases the fibroblast pool. ${ }^{4}$ Secondly, bone marrowderived circulating fibrocytes trafficking to the lung may serve as progenitors for interstitial fibroblasts. ${ }^{5-9}$ Thirdly, alveolar epithelial type II
(ATII) cells, via the process of epithelial-mesenchymal transition (EMT), can undergo a phenotypic, reversible switching to fibroblast-like cells. ${ }^{10-12}$

The orchestrated series of events during EMT includes remodelling of epithelial cell-cell and cellmatrix adhesion contacts, reorganisation of the actin cytoskeleton, induction of mesenchymal gene expression and the acquisition of motile capacity. ${ }^{13}$ During development and disease pathogenesis, EMT is under tight transcriptional control maintained by factors such as Twist, NF- $\mathrm{B}$, Rho, Rac, Snai or GSK-3 $\beta .^{13}{ }^{14}$ The transcription factors eliciting EMT in IPF are yet to be identified. In this context, the zinc finger transcription factor SNAI1 (also called Snail) and SNAI2 (also called Slug) have been reported to act as regulators of EMT during development and disease, including cancer and organ fibrosis. ${ }^{15} 16$

Transforming growth factor $\beta 1$ (TGF $\beta 1$ ) represents a main inducer and regulator of EMT in multiple organ systems, including the lung. ${ }^{10} 17$ While the ability of TGF $\beta 1$ to induce EMT has recently been described in ATII cells in vitro ${ }^{11}{ }^{18-22}$ and in vivo in transgenic mice, ${ }^{12}$ the molecular mechanisms that control these dynamics and their precise role in other experimental models of lung fibrosis and IPF remain to be elucidated.

This study was performed to examine the hypothesis that TGF $\beta 1$ induces EMT via SNAI transcription factors in ATII cells and that SNAI transcription factors contribute to the development of IPF. In detail, we examined the characteristics of TGF $\beta 1$-induced EMT, modulated via SNAI transcription factor in vitro, and evaluated the expression pattern of SNAI transcription factors in experimental as well as human IPF in vivo.

\section{MATERIALS AND METHODS \\ Reagents}

The following antibodies were used in this study: anti-prosurfactant protein (proSP-C) (Chemicon International, Temecula, California, USA), antiaSMA (Sigma, St Louis, Missouri, USA), anti-ecadherin (ECAD) (BD Biosciences, San Jose, California, USA), anti-tight junction protein (TJP)1, anti-occludin (OCCL) (Zymed Laboratories, San Francisco, Calkifornia, USA), anti-SNAI1 and anti- $\alpha$-tubulin (Santa Cruz Biotechnology, Santa Cruz, California, USA), anti-SNAI2 (Cell Signaling Technology, Beverly, Massachusetts, USA) and anti-SNAI1 (a gift from Dr Becker, Institute of Pathology, Technical University of Munich, Germany). Recombinant human TGF $\beta 1$ was purchased from R\&D Systems (Minneapolis, Minnesota, USA). 


\section{Cell culture}

The human lung epithelial cell line A549 (ATCC CCL-185; Manassas, Virginia, USA) was maintained in Dulbecco's modified Eagle's medium (DMEM; Invitrogen, Pasching, Austria), supplemented with $10 \%$ fetal bovine serum (FBS; PAA Laboratories, Carlsbad, California, USA). Primary mouse ATII cells were isolated from adult male C57BL/6N mice as previously described. ${ }^{23}$

\section{Human tissues}

Lung tissue biopsies were obtained from 12 patients with IPF (mean age (SD) 51.3 (11.4) years; six females, six males) and nine transplant donors (serving as control subjects) (mean age 47.5 (13.9) years; four females, five males). ${ }^{24}$ The study protocol was approved by the Ethics Committee of the Justus-LiebigUniversity School of Medicine (AZ 31/93). Informed consent was obtained from each subject for the study protocol.

\section{Animal tissues}

All animal studies were performed in accordance with the guidelines of the Ethic's Committee of University of Giessen School of Medicine and approved by the local authorities. Adult male $\mathrm{C} 57 \mathrm{BL} / 6 \mathrm{~N}$ mice were treated with bleomycin and were sacrificed at the indicated time points.

\section{RNA extraction and reverse transcription-PCR (RT-PCR)}

Total RNA was extracted using RNeasy columns (Qiagen, Hilden, Germany) according to the manufacturer's protocol, and PCR amplification was performed with human and mouse primers (Tables S1 and S2, online supplement).

\section{Quantitative RT-PCR (qRT-PCR)}

qRT-PCR was performed as previously described. ${ }^{23}$ All results were normalised to the relative expression of the constitutively expressed gene porphobilinogen deaminase. The relative transcript abundance of the target gene is expressed in $\Delta \mathrm{Ct}$ values $(\Delta \mathrm{Ct}=\mathrm{Ct}$ reference-Ct target). Relative changes in transcript levels compared with controls are expressed as $\Delta \Delta \mathrm{Ct}$ values $(\Delta \Delta \mathrm{Ct}=\Delta \mathrm{Ct}$ treated $-\Delta \mathrm{Ct}$ control). All $\Delta \Delta \mathrm{Ct}$ values correspond approximately to the binary logarithm of the fold change. The human and mouse primer sequences are depicted in Table S3 and S4, online supplement, respectively.

\section{Small interfering RNA (siRNA) transfection}

The siRNA oligonucleotides specific for human SNAI1 and SNAI2 mRNA (table 1) were obtained from Dharmacon Inc. (Lafayette, Indiana, USA). A549 cells were transiently transfected with $100 \mathrm{nM}$ SNAI or non-specific siRNA using Lipofectamine2000 reagent (Invitrogen). At $4 \mathrm{~h}$ post-transfection, cells were treated with TGF $\beta 1$. Cells were lysed after $24 \mathrm{~h}$ and the efficiency of gene knock-down was monitored.

Table 1 Small interfering RNA (siRNA) sequences

\begin{tabular}{lll}
\hline Gene name & Sense sequence & Antisense sequence \\
\hline SNAI1-si\#1 & ACUCAGAUGUCAAGAAGUAUU & PUACUUCUUGACAUCUGAGUUU \\
SNAI1-si\#2 & GCAAAUACUGCAACAAGGAUU & PUCCUUGUUGCAGUAUUUGCUU \\
SNAI1-si\#3 & GCUCGGACCUUCUCCCGAAUU & PUUCGGGAGAAGGUCCGAGCUU \\
SNAI1-si\#4 & GCUUGGGCAAGUGCCCAAUU & PUUGGGCACUUGGCCCAAGCUU \\
SNAI2-si\#1 & GGACACACAUACAGUGAUUUU & PAAUCACUGUAUGUGUGUCCUU \\
SNAI2-si\#2 & UAAAUACUGUGACAAGGAAUU & PUUCCUUGUCACAGUAUUUAUU \\
SNAI2-si\#3 & GAAUGUCUCUCCUGCACAAUU & PUUGUGCAGGAGAGACAUUCUU \\
SNAI2-si\#4 & GAAUCUGGCUGCUGUGUAGUU & PCUACACAGCAGCCAGAUUCUU \\
\hline
\end{tabular}

\section{Migration assay}

Cell migration was determined using Boyden chamber assay (ThinCerts Tissue Culture Inserts, 24-well, pore size $3.0 \mu \mathrm{m}$ from Kremsmunster, Austria), as described previously. ${ }^{25}$ A549 cells were transfected with SNAI1, SNAI2 or non-specific siRNA at a total concentration of $75 \mathrm{nM}$, detached, and $5 \times 10^{4}$ cells were seeded into the Boyden chamber insert. Cells were cultured for $8 \mathrm{~h}$ to allow their attachment to the membrane and migration was induced by adding TGF $\beta 1(2 \mathrm{ng} / \mathrm{ml})$ to the medium in the lower wells. After $24 \mathrm{~h}$, cells were fixed and stained using crystal violet solution, and non-migrated cells were removed by cotton swabbing. The membranes were carefully separated from the insert wall, and optical densities of migrated cells were measured with a GS-800 Calibrated Densitometer and analysed with Quantity One software.

\section{Statistical analysis of data}

Values are presented as the mean (SEM). All $\Delta \mathrm{Ct}$ values obtained from qRT-PCR were analysed for normal distribution using the Shapiro-Wilk test, with the assignment of a normal distribution with $p>0.05$. All $\Delta \Delta$ Ct values were analysed using the two-tailed, one-sample $t$ test. Normality of data was confirmed using quantile-quantile plots. Intergroup differences of $\Delta \mathrm{Ct}$ values from patients and bleomycin-treated mice were derived using a one-tailed, two-sample $t$ test. A one-way analysis of variance (ANOVA) with Tukey HSD post hoc test was for studies with more that two groups. A level of $p<0.05$ was considered statistically significant.

\section{Further experimental procedures}

The detailed procedures for western blotting, densitometric analysis, immunohistochemistry, immunofluorescence, laserassisted microdissection and transfection of human SNAI1 and SNAI2, as well as the sequences of all primers used in this study, are provided in the online supplement.

\section{RESULTS}

\section{TGFB1-induced EMT in ATII cells}

Initially, a comprehensive analysis of TGF $\beta 1$-induced EMT in ATII cells was performed. We assessed the gene and protein expression as well as the localisation of epithelial and mesenchymal markers in primary mouse ATII cells (Supplementary fig E1) and the human ATII cell line A549. TGF $\beta 1$ treatment for $24 \mathrm{~h}$ induced changes in $\alpha \mathrm{SMA}$, ECAD and TJP1 expression and/or localisation, indicative of EMT. While ECAD and TJP1 staining decreased, an appreciable number of cells expressing aSMA increased from 5.9\% (2.8\%) in vehicle-treated, to $28.2 \%(8.1 \%)$ in TGF $\beta 1$-treated cells (fig 1A,B). The presence of $\alpha \mathrm{SMA}$ and TJP1 double-positive cells in TGF- $\beta 1$-treated, but not in vehicle-treated cells, further corroborated the occurrence of TGF $\beta 1$-induced EMT in primary ATII cells (fig 1A, bottom panels).

\section{SNAI transcriptions factor expression in TGF $\beta 1$-induced EMT in ATII cells}

We subsequently quantified the mRNA expression patterns of several EMT marker genes in ATII cells, using qRT-PCR. ATII cells were treated with TGF $\beta 1$ in a dose- and time-dependent manner as indicated (fig 2, Supplementary fig E2). Although the magnitude of EMT marker gene expression in both cell types investigated differs, most markers were differentially regulated after $8 \mathrm{~h}$. TGF $\beta 1$ treatment led to a decreased expression of the epithelial cell marker ECAD and occludin (OCCL), concomitant 
Figure 1 Transforming growth factor $\beta 1$ (TGF $\beta 1$ )-induced epithelial-mesenchymal transition (EMT) in alveolar epithelial type II (ATII) cells. (A) Immunofluorescence detection of $\alpha$-smooth muscle actin ( $\alpha S M A)$, e-cadherin (ECAD) and tight junction protein 1 (TJP1) was performed after treatment with TGF $\beta 1(2 \mathrm{ng} / \mathrm{ml})$ or vehicle for $24 \mathrm{~h}$. Co-localisation of $\alpha \mathrm{SMA}$ (red) and TJP1 (green) was assessed by immunofluorescence in ATIl cells treated with TGF $\beta 1$ for 24 h. Nuclei are visible by 4'6-diamidino-2-phenylindole (DAPI) staining. Original magnification is $\times 40$. (B) The quantification of the percentage of aSMA-positive cells $24 \mathrm{~h}$ after TGF $\beta 1$ (2 ng/ml) treatment. The original magnification of a representative figure showing immunofluorescence detection of $\alpha$ SMA (green) with TGF $\beta 1(2 \mathrm{ng} / \mathrm{ml}$ ) or vehicle for $24 \mathrm{~h}$ is $\times 10$. Results are representative of 10 independent experiments and data are expressed as mean (SEM); ${ }^{*} \mathrm{p}<0.05, \mathrm{n}=10$.
A

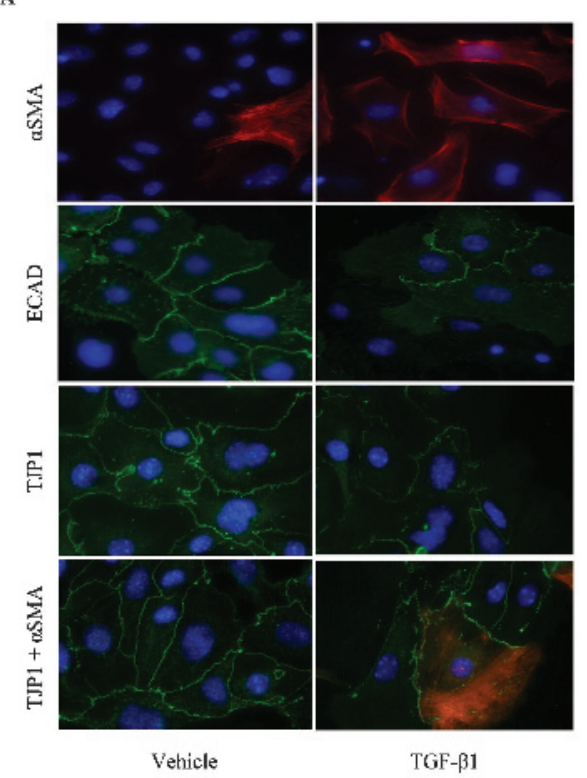

B
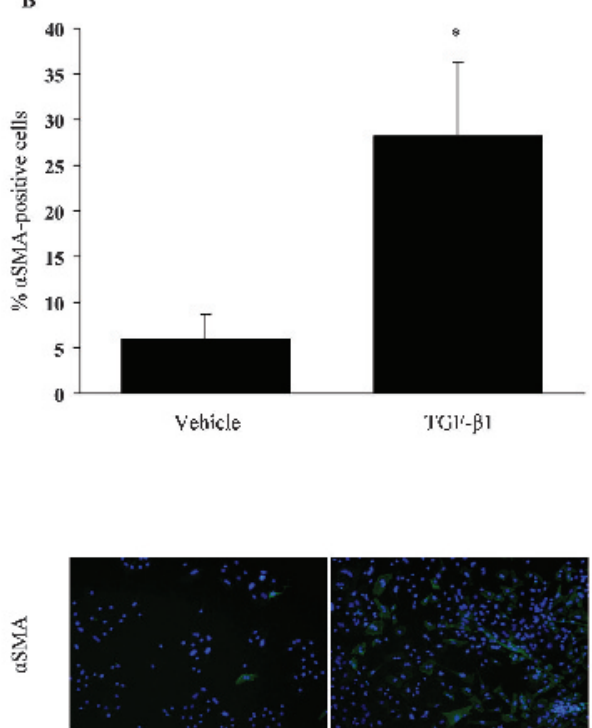

Vehicle

TGF- $\beta 1$
A

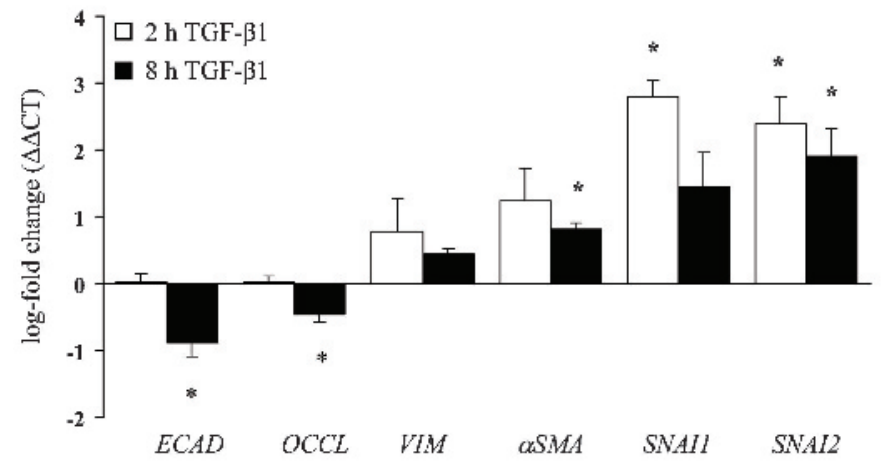

A549 cells

C

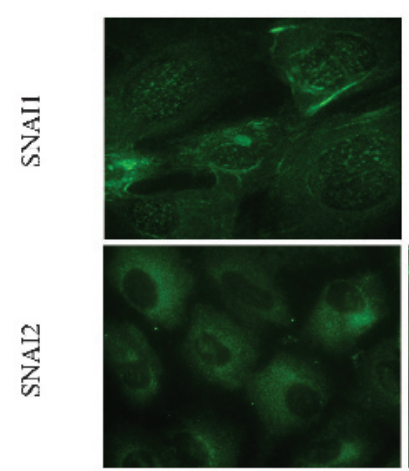

Vehicle
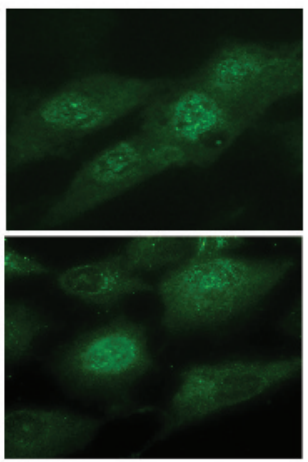

TGF- $\beta 1$

B

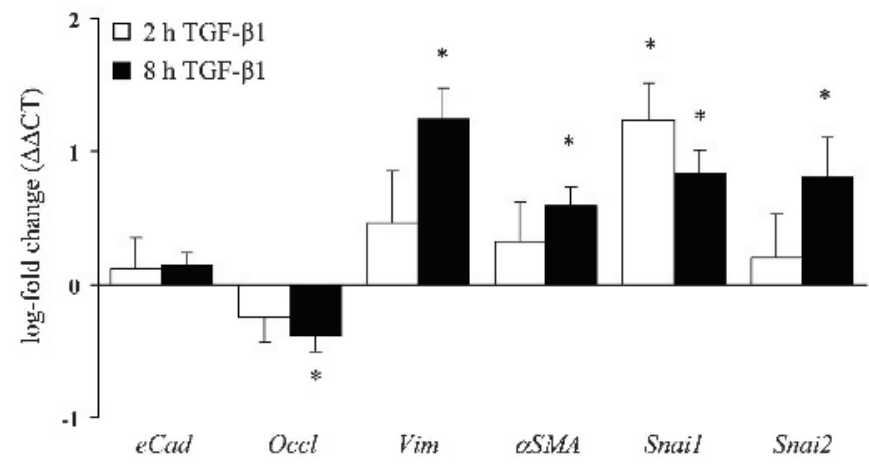

ATII cells

D

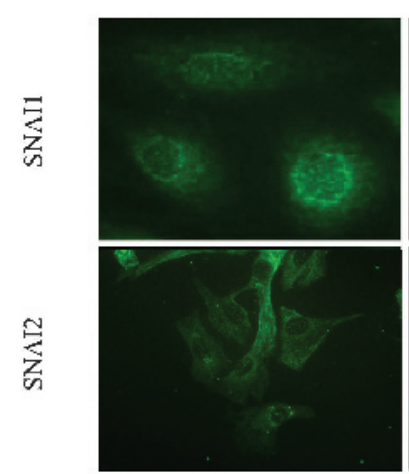

Vehicle

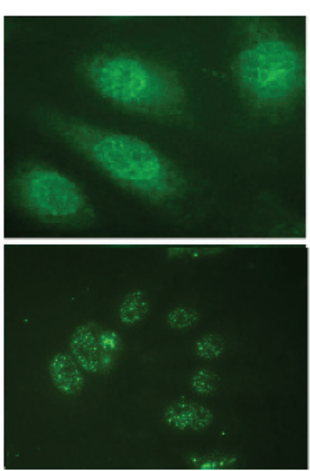

TGF- $\beta 1$

Figure 2 SNAl transcription factor expression in transforming growth factor $\beta 1$ (TGF $\beta 1$ )-induced epithelial-mesenchymal transition (EMT) in alveolar epithelial type II (ATII) cells. Using quantitative reverse transcription-PCR analysis, the expression pattern of the indicated EMT markers was detected in A549 (A) and primary mouse ATII cells (B), after TGF $\beta 1(2 \mathrm{ng} / \mathrm{ml})$ treatment for 2 and $8 \mathrm{~h}$, as indicated. Data are expressed as the mean (SEM); ${ }^{*} \mathrm{p}<0.05, n=5$. ( $C$ and D) Immunofluorescence analysis was performed using a primary antibody directed against SNAI1 and SNAI2 in both cells. The original magnification of the representative image from three independent experiments is $\times 40$. ECAD, e-cadherin; OCCL, occludin; $\alpha S M A, \alpha$-smooth muscle actin; VIM, vimentin. 
with increased expression of the mesenchymal marker vimentin $(V I M)$ and $\alpha S M A, 8 \mathrm{~h}$ after treatment of ATII cells. Notably, VIM was strongly induced in primary ATII cells, but not in A549 cells (fig 2A,B). Importantly, we observed a significant upregulation of both SNAI1 and SNAI2, along with the alterations in EMT marker gene expression (fig 2A,B).

Since the SNAI transcription factors were regulated at the mRNA level, we next wanted to assess the protein expression and function in TGFß1-induced EMT in ATII cells. Immunofluorescence analysis of TGF $\beta 1$-treated ATII cells revealed increased nuclear translocation of endogenous SNAI1 and SNAI2 upon TGF $\beta 1$ treatment in A549 and primary ATII cells, respectively (fig 2C,D, Supplementary fig E3). In addition, SNAI1 and SNAI2 protein expression was increased by TGF $\beta 1$ in a time-dependent manner, as assessed by western blot analysis. These changes were in concert with the timedependent decrease of ECAD and increased $\alpha \mathrm{SMA}$ expression in response to TGF $\beta 1$ treatment (fig $3 \mathrm{~A}, \mathrm{~B}$ ).

\section{EMT induction by SNAI transcription factor overexpression in ATII cells}

To investigate further whether SNAI has a functional role in inducing EMT in the lung, the full-length SNAI1 and SNAI2 cDNA were cloned into mammalian expression vectors and transiently transfected into A549 cells (transfection efficiency is depicted in Suppplementray fig E4). We examined the gene and protein expression pattern of different EMT marker post-SNAI

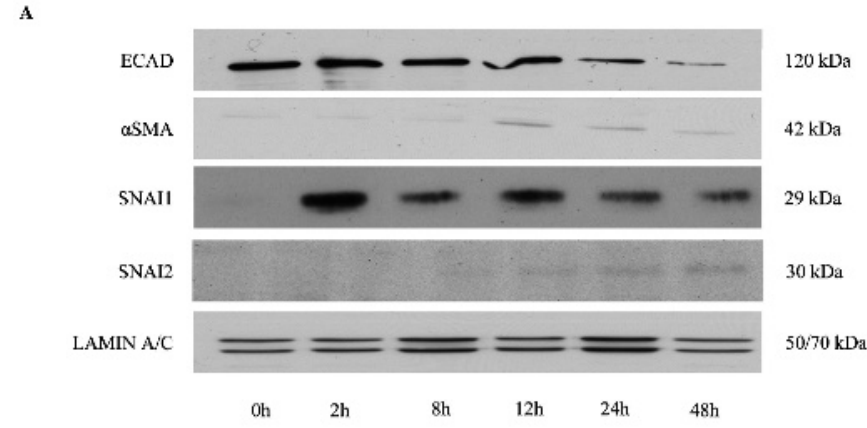

B

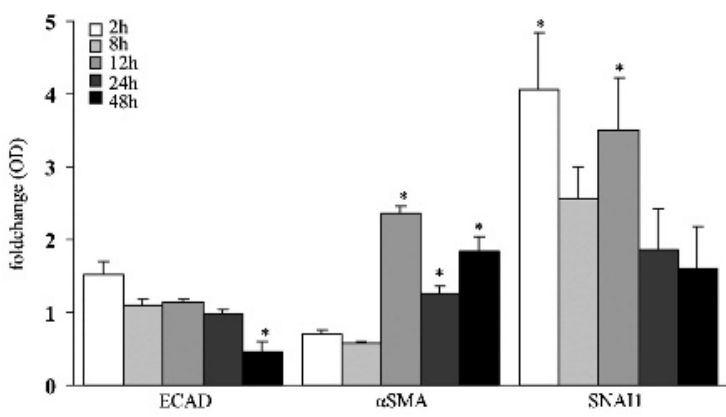

Figure 3 Protein expression of epithelial-mesenchymal transition (EMT) markers in transforming growth factor $\beta 1$ (TGF $\beta 1$ )-treated alveolar epithelial type II (ATII) cells. Protein expression of e-cadherin (ECAD), $\alpha$ smooth muscle actin ( $\alpha$ SMA), SNAI1 and SNAI2 was assessed by western blot analysis. A549 cells were treated with TGF $\beta 1$ (2 ng/ml) for several different times as indicated. Western blot analysis $(A)$ was performed using primary antibody directed against ECAD, aSMA, SNAI1 and SNAI2. Lamin A/C served as a loading control. (B) Western blots were scanned and intensity is represented as fold change. Data are representative of three independent experiments and are expressed as the mean (SEM); ${ }^{*} \mathrm{p}<0.05, \mathrm{n}=3$. transfection. SNAI1 overexpression led to a significantly increased $\alpha \mathrm{SMA}$ expression (fig 4A), which was further confirmed on the protein level (fig 4B, Supplementary fig E5A). Interestingly, SNAI2 overexpression revealed significant changes on the gene and protein level of the epithelial cell targets ECAD and OCCL; however, no significant changes were observed for $\alpha$ SMA expression (fig 4C,D and Supplementary fig E5B). Taken together, overexpression of SNAI transcription factors is able to induce EMT in ATII cells, even in the absence of TGF $\beta 1$.

\section{Effect of SNAI transcription factor silencing on TGF $\beta 1$-induced EMT in ATII cells}

In order to determine the effect of SNAI1 or SNAI2 on the process of TGF $\beta 1$-induced EMT in ATII cells, we next characterised SNAI mRNA knock-down using four different sequences of siRNA oligonucleotides targeting SNAI1 and SNAI2, respectively (Supplementary fig E6). Quantitative and semi-quantitative RT-PCR analysis revealed that three out of four SNAI1 (Supplementary fig E6A, B) and SNAI2 (Supplementary fig E6C, D) siRNA oligonucleotides were effective in reducing the respective mRNA level in response to TGF $\beta 1$ treatment. The most effective siRNA oligonucleotides targeting SNAI1 and SNAI2 were then used to examine the effect of SNAI depletion on TGF $\beta 1$-induced EMT. Both SNAI1 and SNAI2 depletion effectively attenuated TGF- $\beta 1$-induced EMT in ATII cells (fig 5). SNAI1-depleted ATII cells exhibited a significantly attenuated decrease in OCCL and TJP1, as well as a reduced increase in $\alpha S M A$ mRNA levels, as assessed by qRTPCR and semi-quantitative RT-PCR $24 \mathrm{~h}$ after TGF $\beta 1$ treatment compared with non-specific scrambled siRNA-treated ATII cells (fig 5A,B). Similarly, SNAI2 depletion in ATII cells demonstrated an increase in OCCL and TJP1 mRNA level compared with TGF $\beta 1$-treated non-specific scrambled siRNAdepleted ATII cells, whereas no significant changes have been observed in mesenchymal marker expression (fig 5C,D).

\section{Effect of SNAI transcription factor silencing on TGF $\beta 1$-induced migration in ATII cells}

One of the key requirements for the complete process of EMT is the capability of ATII cells to migrate. TGF $\beta 1$ induces ATII cell migration in a dose-dependent manner (Supplementary fig E7). In order to elucidate the impact of SNAI transcription factor on TGF $\beta 1$-induced ATII cell migration, we performed an in vitro migration assay on SNAI1- and SNAI2-depleted cells stimulated with TGF $\beta 1$. After $24 \mathrm{~h}$ of TGF $\beta 1$ treatment, cells transfected with non-specific siRNA exhibited a fourfold induction of cell migration (fig 6). Both SNAI1- and SNAI2-depleted cells exhibited significant reduced migration under baseline and TGF $\beta 1$-induced conditions (fig 6).

\section{SNAI transcription factor expression in experimental lung fibrosis}

To analyse whether expression of SNAI transcription factors was regulated during experimental lung fibrosis, we subjected mice to bleomycin treatment for 7 or 14 days. On the mRNA level, a significant increase in SNAI1 has been observed in lung homogenates from bleomycin-treated mice compared with saline-treated mice, as assessed by qRT-PCR analysis (fig 7A). Interestingly, significantly elevated levels of both SNAI1 and SNAI2 were observed in freshly isolated ATII cells from bleomycin-treated lungs compared with ATII cells from salinetreated lungs, as early as 7 days after treatment (fig 7B). Using 
A

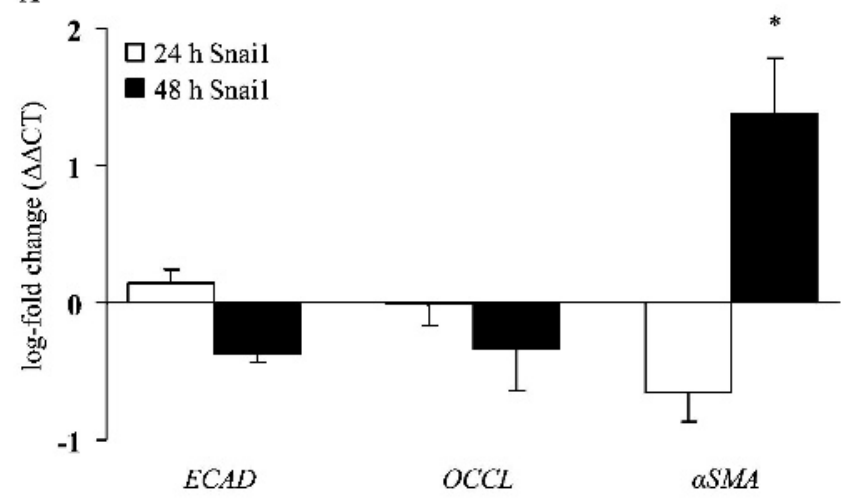

C

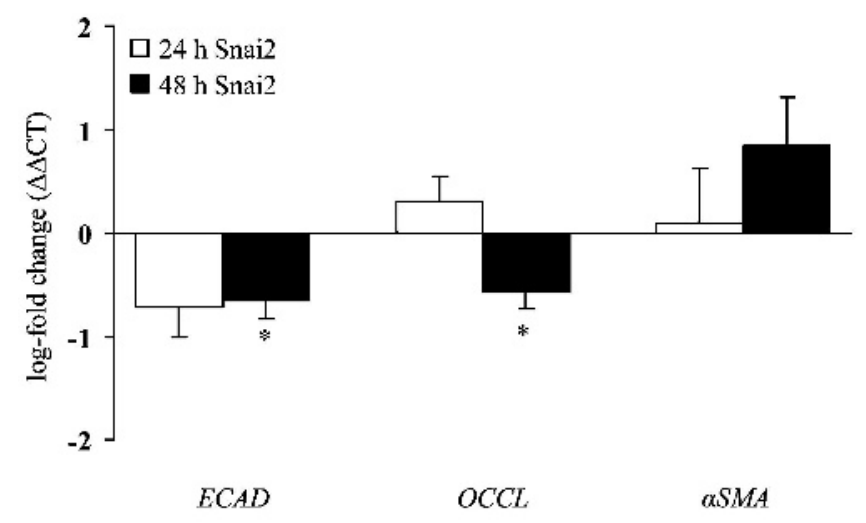

B

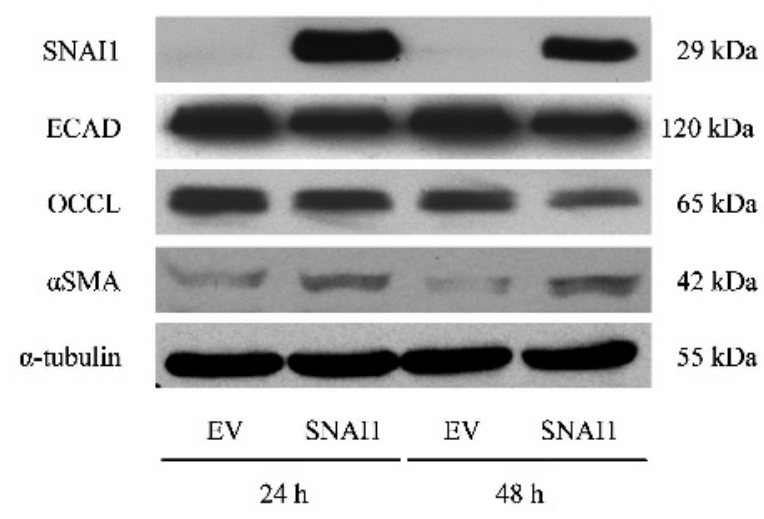

D

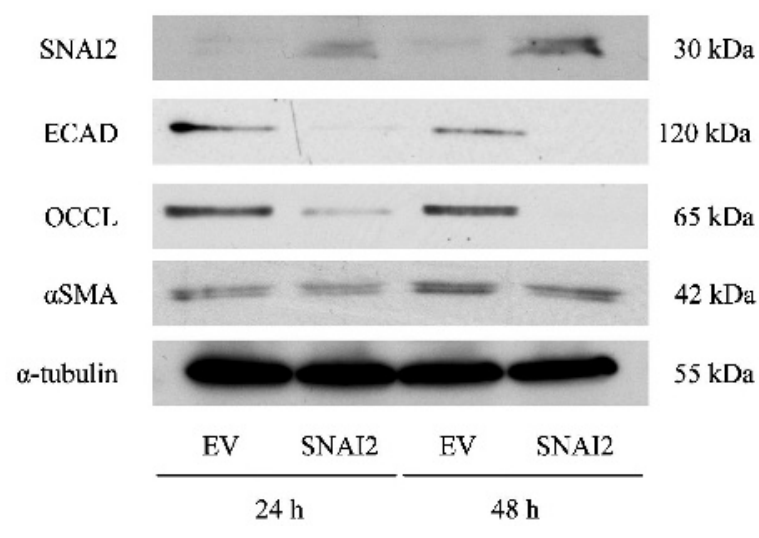

Figure 4 Epithelial-mesenchymal transition (EMT) induction by SNAI transcription factor overexpression in alveolar epithelial type II (ATII) cells. EMT marker expression was analysed by quantitative reverse transcription-PCR (A, C) and western blot (B, D). A549 cells overexpressing human SNAI1 (A, B) or SNAI2 (C, D) protein for 24 and $48 \mathrm{~h}$; analysis was carried out by comparison with the empty vector (EV) control. The $\alpha$-tubulin served as the loading control. Data are representative of five independent experiments and are expressed as the mean (SEM); ${ }^{*} p<0.05, n=5$. ECAD, e-cadherin; OCCL, occludin; $\alpha$ SMA, $\alpha$-smooth muscle actin.

immunohistochemistry, we next determined the localisation of SNAI1 and SNAI2 in bleomycin-treated lungs. While SNAI1 was predominantly localised to perivascular mesenchymal cells and alveolar macrophages in saline-treated lungs, it also demonstrated localisation in the epithelium and, particularly, in subepithelial areas, after bleomycin treatment (fig 7C). Although little or no staining for SNAI2 was detectable in saline-treated lungs, weak SNAI2 staining localised to the lung interstitium after bleomycin treatment (fig 7C). $\alpha$ SMA served as a well-characterised marker of the lung interstitium after bleomycin treatment.

\section{SNAI transcription factor expression in IPF}

Finally, we asked whether SNAI expression was also increased in the lungs of patients with IPF. To this end, we determined the mRNA expression in lung homogenates obtained from patients with IPF and transplant donors. The qRT-PCR analysis revealed increased levels of SNAI2 gene expression in both lung homogenates and microdissected alveolar septae from patients with IPF compared with transplant donors (fig 8A,B). Immunohistochemistry further confirmed the expression and localisation of SNAI1 and SNAI2 predominantly in hyperplastic alveolar epithelial cells in IPF, whereas only weak SNAI1 and SNAI2 expression was detected in lung specimens of transplant donors (fig 8C). Western blot analysis further corroborated the elevated protein level of both SNAI1 and SNAI2 in lung homogenates from patients with IPF compared with transplant donors (fig 8D).

\section{DISCUSSION}

IPF is the most common form of idiopathic interstitial pneumonias (IIPs), which exhibits a poor prognosis and unresponsiveness to currently available therapies, reflecting our limited understanding of the basic mechanisms and mediators implicated in the pathogenesis of this disease. ${ }^{12627}$ While the initial injury in IPF is affecting the alveolar epithelium, the interstitial fibroblast/activated (myo)fibroblast represents the key effector cell accumulating in fibroblast foci and responsible for the increased extracellular matrix deposition that is characteristic for IPF. ${ }^{28-30}$ The EMT, a process where alveolar epithelial cells turn into fibroblast-like cells, has recently been reported to serve as a source for the fibroblast pool in tissue fibrosis of the liver, kidney and lung. ${ }^{11} 1231$ EMT was initially described in embryonic development. ${ }^{14}$ The orchestrated series of events during EMT involves changes in cell polarity, loss of epithelial cell markers, induction of mesenchymal gene expression and enhanced cell migration. ${ }^{13}$ TGF $\beta 1$, known as a key profibrotic growth factor, has emerged 
A

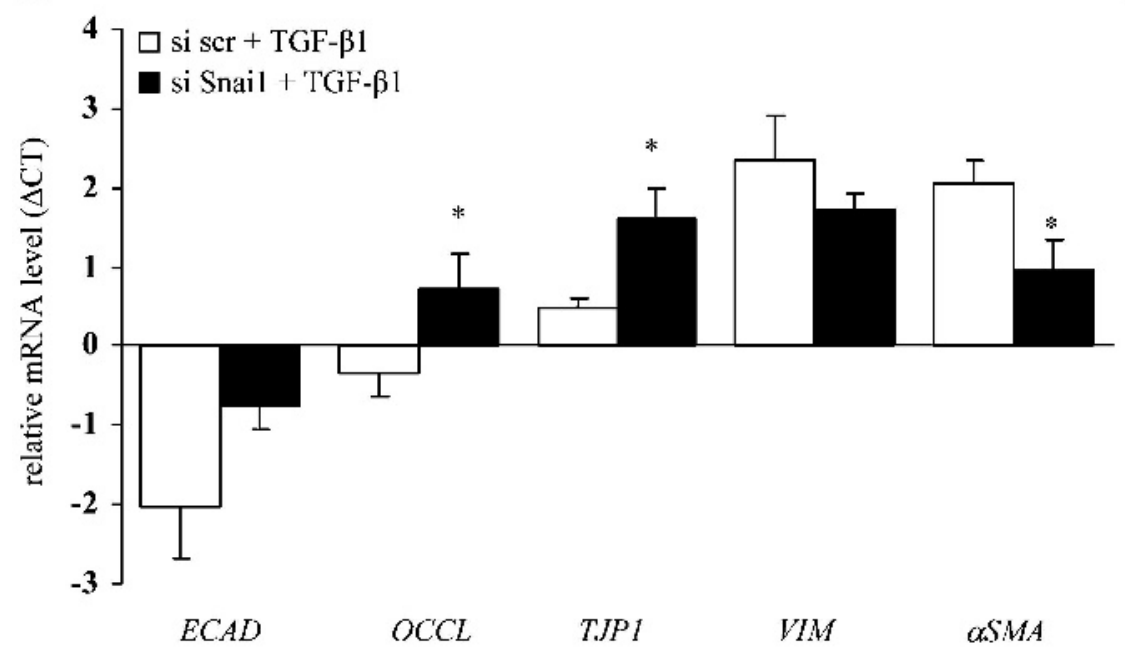

B

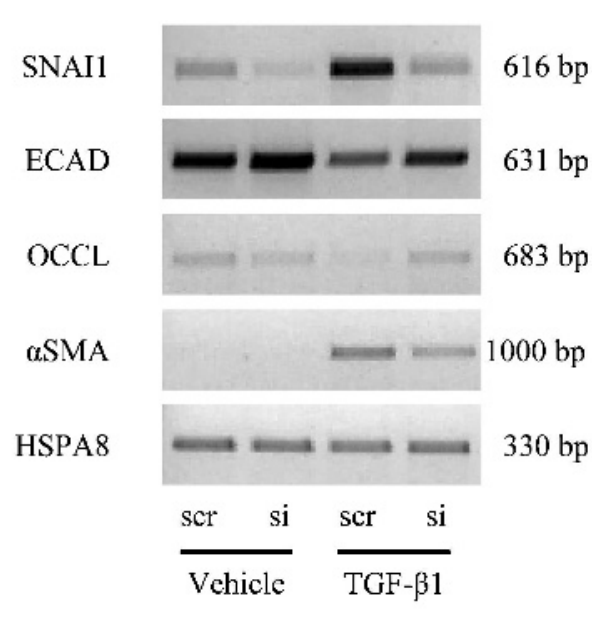

D

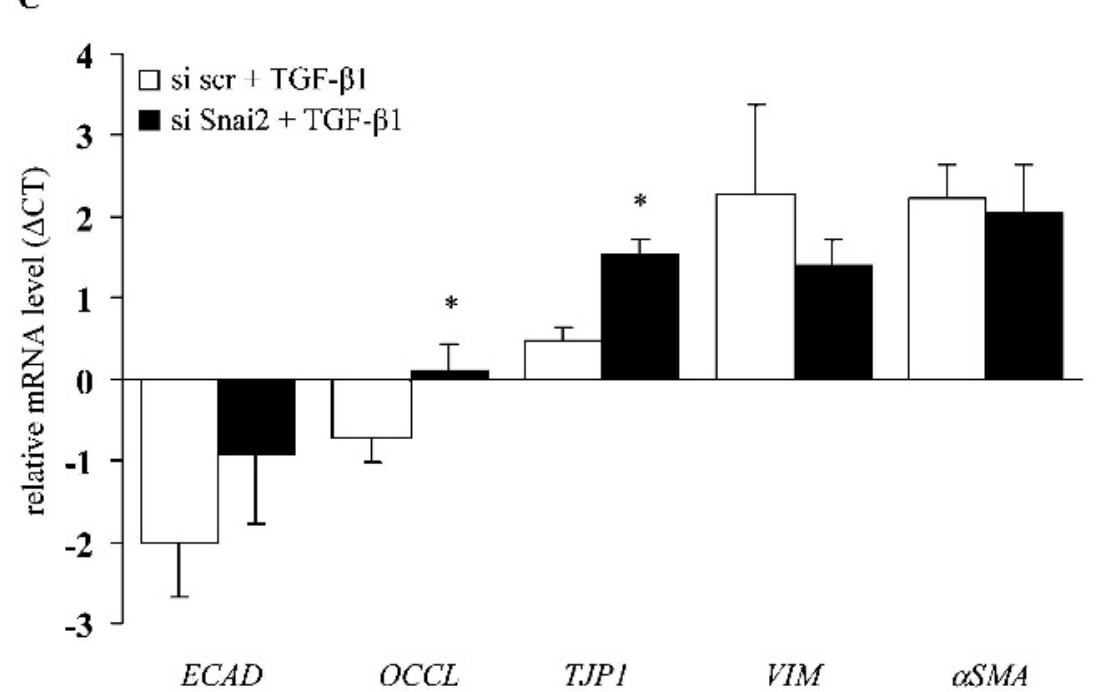

SNAI2

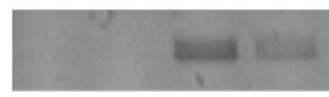

$631 b p$

ECAD

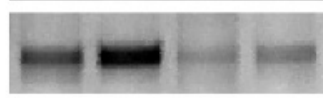

$631 b p$

OCCL

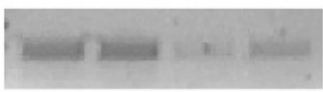

683 bp

$\alpha \mathrm{SMA}$

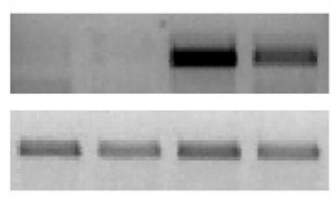

$1000 \mathrm{bp}$

HSPA8

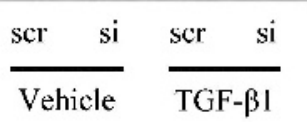

Figure 5 Effect of SNAI transcription factor silencing on transforming growth factor $\beta 1$ (TGF $\beta 1$ )-induced epithelial-mesenchymal transition in alveolar epithelial type II (ATII) cells. (A, C) Using quantitative reverse transcription-PCR (RT-PCR) analysis, the expression patterns of EMT marker genes was assessed in A549 cells after treatment with small interfering RNA (siRNA) against SNAI1 (A) and SNAI2 (C), with TGF $\beta 1$ exposures for 24 h and compared with control non-specific scrambled siRNA treatment with TGF $\beta 1$ exposures for $24 \mathrm{~h}$. (B, D) Semi-quantitative RT-PCR analysis; the expression patterns of EMT marker genes was detected in A549 cells after siRNA treatment against SNAI1 (B) and SNAI2 (D), with TGF $\beta 1$ exposures for $24 \mathrm{~h}$ (lane 4) and compared with controls (1) non-specific scrambled siRNA treatment with (lane 3) or without (lane 1) TGF $\beta 1$ for $24 \mathrm{~h}$ and (2) siRNA against SNAI without TGF $\beta 1$ treatment (lane2). HSPA8 (heat shock $70 \mathrm{kDa}$ protein 8) was employed as loading control. Data are representative of three independent experiments and are expressed as mean (SEM); ${ }^{*} \mathrm{p}<0.05, n=3$. ECAD, e-cadherin; OCCL, occludin; scr; scrambled siRNA oligonucleotide; $\alpha \mathrm{SMA}, \alpha$-smooth muscle actin; TJP1, tight junction protein 1 ; VIM, vimentin.

as a potent inducer of EMT. ${ }^{17}{ }^{32}$ In the present study, we report that SNAI transcription factors are key regulators of TGF $\beta 1$ induced EMT in the lung. Elevated expression of SNAI1 and SNAI2 was observed in ATII cells in response to TGF $\beta 1$ in vitro. Depletion of SNAI1 and SNAI2 using siRNA knock-down in ATII cells inhibited TGF $\beta 1$-induced alterations in EMT marker gene expression and ATII cell migration in response to TGF $\beta 1$. Interestingly, ectopic overexpression of SNAI transcription factors promotes EMT even in the absence of TGF $\beta 1$. Finally, an increased level of SNAI1 and SNAI2 in experimental and human IPF in vivo further indicated a significant contribution of SNAI transcriptions factors to the process of EMT in lung fibrosis.

At the onset of our studies, we performed a detailed analysis of the occurrence of EMT in ATII cells in response to TGF $\beta 1$, determining marker gene and protein expression and localisation in primary mouse ATII cells and A549 cells. Upon exposure to TGF $\beta 1$, ATII cells demonstrated an increased expression of mesenchymal markers (such as $\alpha \mathrm{SMA}$ ) with a corresponding decrease in epithelial markers (ECAD and TJP1), suggestive of EMT. EMT was further corroborated by the presence of $\alpha \mathrm{SMA}$ and TJP1 double-positive cells in TGF $\beta 1$-treated, but not in untreated cells. These results are in accordance with previous studies, reporting that TGF $\beta 1$ induces EMT in lung epithelial cells in vitro. ${ }^{11}{ }^{19-21}$ Recently, TGF $\beta 1$-induced EMT has also been demonstrated in vivo in a triple transgenic mouse model. ${ }^{12}$

Several regulatory molecules have been implicated in the process of EMT. ${ }^{13}{ }^{17}$ Here, we demonstrated increased expression and nuclear translocation of the zinc finger transcription 


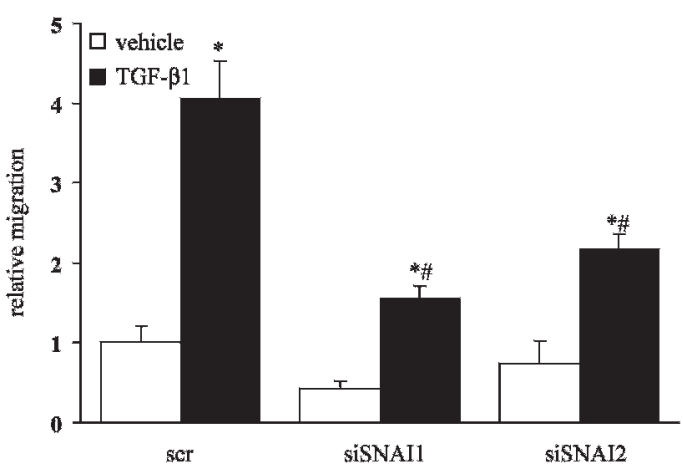

Figure 6 Effect of SNAI transcription factor silencing on transforming growth factor $\beta 1$ (TGF $\beta 1$ )-induced migration in alveolar epithelial type II (ATII) cells. The small interfering RNA (siRNA)-treated A549 cells were treated or not with TGF $\beta 1$ for $24 \mathrm{~h}$. The relative migration potential was assessed using Boyden chamber assay. scr; scrambled siRNA oligonucleotide. Membranes were scanned and the intensity is represented as bars. Data are representative of three independent experiments and are expressed as mean (SEM); ${ }^{*} p<0.05, n=3$. factors SNAI1 and SNAI2 along with EMT in ATII cells. Elevated levels of SNAI1 mRNA have been reported in TGF $\beta 1$-treated A549 cells, ${ }^{22}$ which has been further confirmed in this study. In addition, SNAI1 induction by TGF $\beta 1$ has been recently demonstrated in MDCK cells, a canine renal epithelial cell line. ${ }^{33}$ Furthermore, it has been demonstrated that SNAI1-deficient mice die at the gastrulation stage, ${ }^{34}$ because of their inability to undergo EMT, reinforcing the importance of the SNAI transcription factors in the process of embryonic development.

The impact of SNAI-mediated EMT in pathophysiological conditions such as cancer or tissue fibrosis, however, is less well substantiated and requires further investigations. Recently, high SNAI expression has been associated with poor prognosis and tumour recurrence in patients with lung cancer. ${ }^{35}$ In addition, SNAI1 has been reported to induce chemoresistance of cancer cells. ${ }^{36}$ In tissue fibrosis, SNAI-mediated EMT has been proposed to be involved in kidney fibrosis. ${ }^{37}$ The comprehensive analysis presented herein strongly suggests that SNAI1 and SNAI2 are essential mediators involved in the initiation and perpetuation of TGF $\beta 1$-mediated EMT in experimental and human IPF, but
A

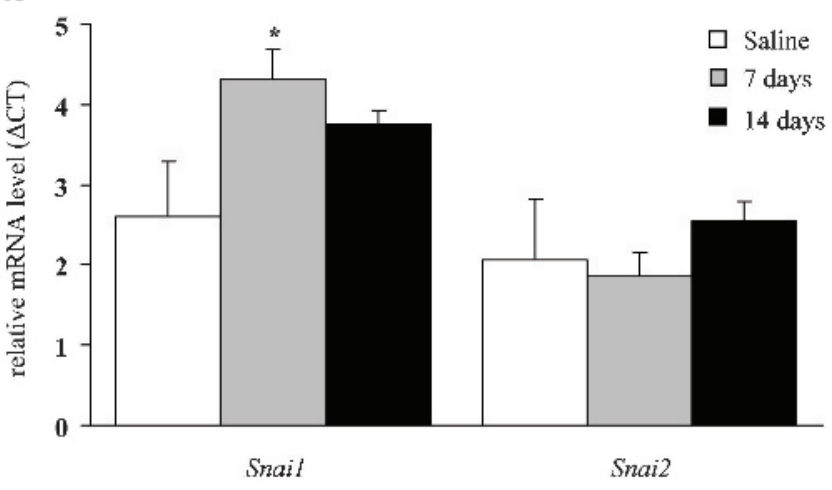

B

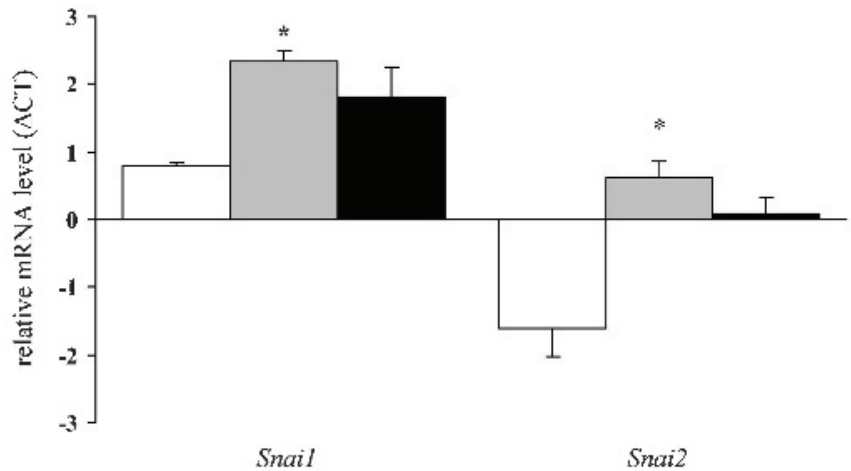

C

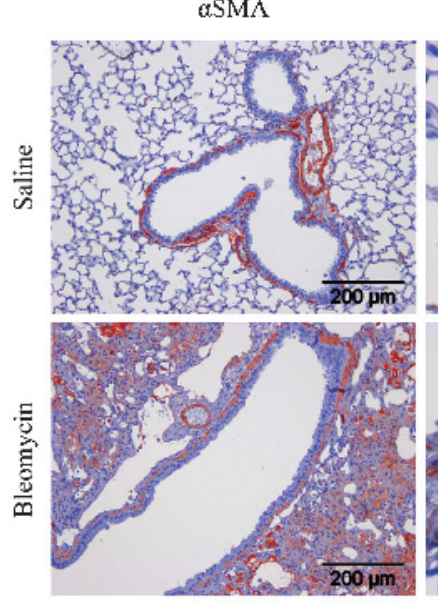

SNAII

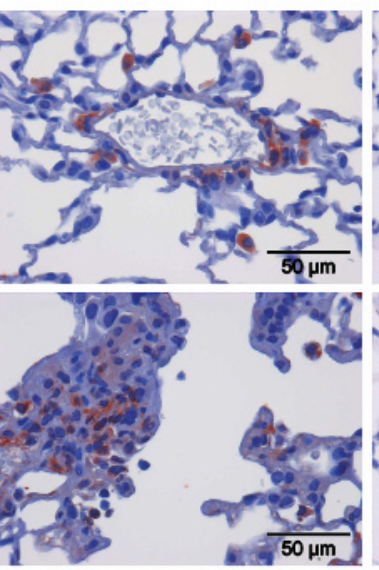

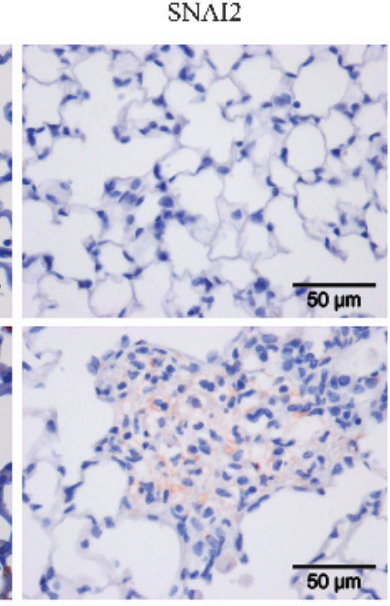

Figure 7 SNAI transcription factor expression in experimental lung fibrosis. Mice were exposed to bleomycin, and lungs were harvested after 7 or 14 days, as indicated. (A) RNA was isolated and quantitative reverse transcription-PCR (qRT-PCR) was performed for SNAl genes in 7 or 14 day bleomycin- or saline-treated lung homogenates. (B) SNAI gene expression was quantified in primary alveolar epithelial type II (ATII) cells freshly isolated from 7 or 14 day bleomycin-treated lungs, using qRT-PCR. Data are representative of five independent experiments and are expressed as mean (SEM); ${ }^{*} p<0.05, n=5$. (C) Immunohistochemical analysis of $\alpha$-smooth muscle actin ( $\alpha$ SMA), SNAl1 and SNAI2 localisation was performed in paraffinembedded tissue from bleomycin- or saline-treated lungs after 14 days. 
A

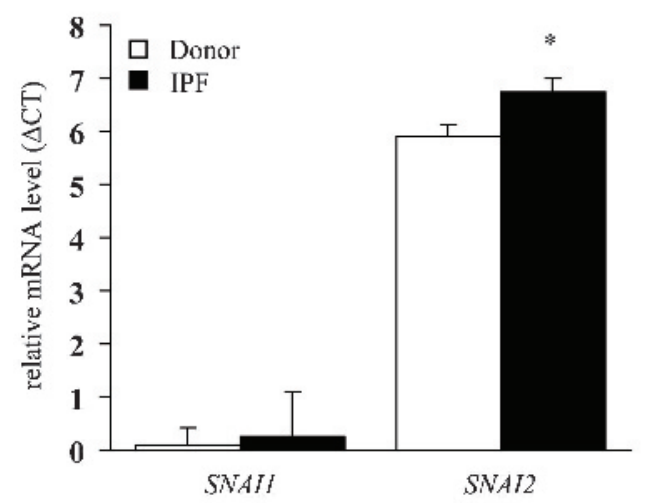

C.

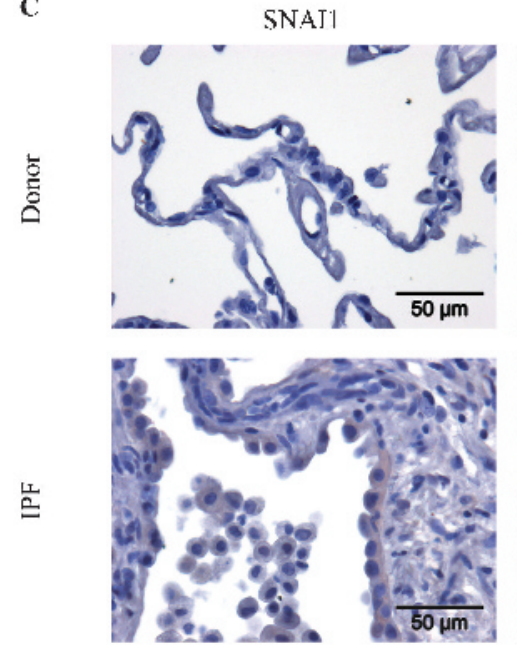

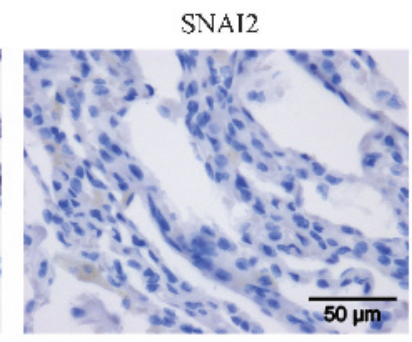

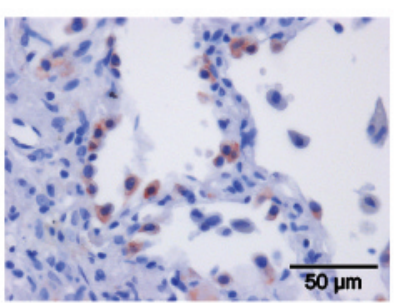

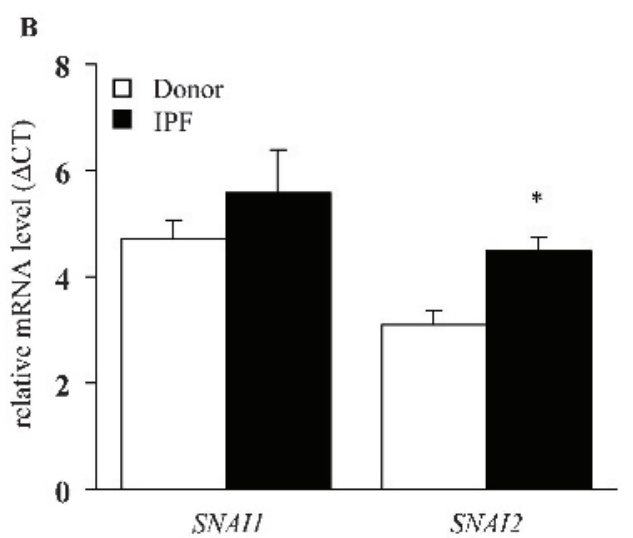

D

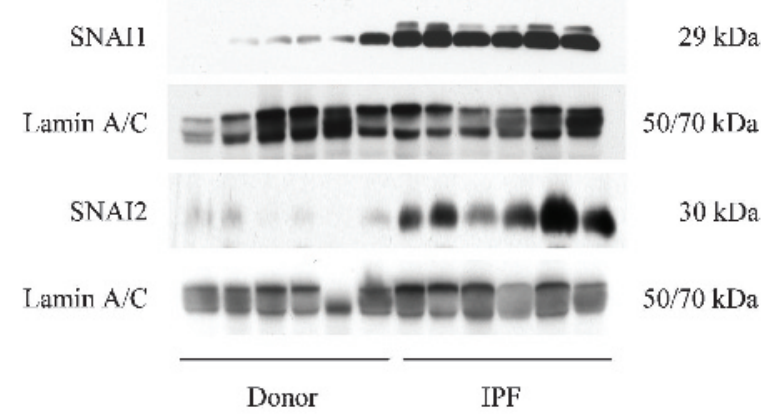

Figure 8 SNAl transcription factor expression in idiopathic pulmoray fibrosis (IPF). (A) Gene expression analysis of SNAI1 and SNAI2 genes was performed by quantitative reverse transcription-PCR (qRT-PCR) analysis of total RNA derived from lung homogenates of 12 patients with IPF and nine transplant donors. (B) SNAI1 and SNAI2 mRNA levels were quantified using qRT-PCR in laser-assisted microdissected septae from five patients with IPF and five transplant donors. Data are expressed as the mean (SEM); ${ }^{*} p<0.05, n=5$. (C) Immunohistochemical analysis of SNAI1 and SNAI2 localisation was performed in a paraffin-embedded lung specimen from patients with IPF or transplant donors. (D) Western blot analysis of SNAI1 and SNAI2 was performed three times in lung homogenates obtained from six patients with IPF and six transplant donors. Lamin A/C served as the loading control.

by no means proof of EMT in IPF in vivo. Since we were able to inhibit EMT in ATII cells with siRNAs targeting SNAI1 and SNAI2, it will have to be demonstrated whether in vivo interference with these factors may lead to an attenuation of fibrosis. We also present evidence that both SNAI1 and SNAI2 induce EMT even in the absence of TGF $\beta 1$, hence recapitulating their role as potent inducers of EMT. It has to be pointed out that SNAI1 and SNAI2 may differ in their respective target genes. Our results suggest that SNAI1 preferably regulated aSMA, whereas SNAI2 alters epithelial targets. This is of special interest, as cell-specific SNAI expression in vivo may lead to distinct cell fates. Given that SNAI2 was the dominant transcription factor regulated in human IPF tissue, we propose that some ATII cells undergoing EMT in IPF may exhibit an aSMA-negative fibroblast phenotype mediated by SNAI2.

In IPF, recent studies were able to demonstrate evidence of EMT in lung tissue biopsies, suggesting that this process contributes to the increased pool of (myo)fibroblasts in lung fibrosis, ${ }^{11}{ }^{12}$ as well as in allografts after lung transplantation. ${ }^{38}$ In contrast, another recent study failed to supply evidence for EMT in pulmonary fibrosis. ${ }^{39}$ This discrepancy may be due to the transient nature and complexity of the EMT process, and further highlights the challenge in this research field.

Taken together, we demonstrated that SNAI transcription factors mediate EMT in ATII cells in vitro and their expression is increased in experimental and human IPF in vivo. We speculate that EMT is an early event in IPF and that activation and nuclear translocation of SNAI transcription factors constitute an important early regulator of EMT in ATII cells.

Acknowledgements: We thank Dr Becker (Institute of Pathology, Technical University of Munich, Germany) for the generous gift of anti-rat monoclonal SNAl1 antibody. We are indebted to all members of the Eickelberg Lab for stimulating discussions, and Andreas Jahn and Simone Becker for excellent technical assistance.

Funding: The authors are supported by the Helmholtz Association, the German Research Foundation (DFG) KliFo 118, the International Graduate Program "Signaling Mechanisms of Lung Physiology and Disease" GRK1062, and a career development award by the University of Giessen School of Medicine to M.K.

Competing interests: None.

Ethics approval: The study protocol was approved by the Ethics Committee of the Justus-Liebig-University School of Medicine (AZ 31/93)

Provenance and peer review: Not commissioned; externally peer reviewed. 


\section{REFERENCES}

1. American Thoracic Society/European Respiratory Society International Multidisciplinary Consensus Classification of the Idiopathic Interstitial Pneumonias. June 2001. Am J Respir Crit Care Med 2002;165:277-304.

2. King TE Jr, Schwarz MI, Brown K, et al. Idiopathic pulmonary fibrosis: relationship between histopathologic features and mortality. Am J Respir Crit Care Med 2001;164:1025-32.

3. Visscher DW, Myers JL. Histologic spectrum of idiopathic interstitial pneumonias Proc Am Thorac Soc 2006;3:322-9.

4. Phan SH. The myofibroblast in pulmonary fibrosis. Chest 2002;122(6 Suppl):286S-89S.

5. Hashimoto $\mathbf{N}$, Jin H, Liu T, et al. Bone marrow-derived progenitor cells in pulmonary fibrosis. J Clin Invest 2004;113:243-52.

6. Phillips RJ, Burdick MD, Hong K, et al. Circulating fibrocytes traffic to the lungs in response to CXCL12 and mediate fibrosis. J Clin Invest 2004;114:438-46.

7. Moeller A, Gilpin SE, Ask K, et al. Circulating fibrocytes are an indicator of poor prognosis in idiopathic pulmonary fibrosis. Am J Respir Crit Care Med 2009;179:588-94.

8. Moore BB, Murray L, Das A, et al. The role of CCL12 in the recruitment of fibrocytes and lung fibrosis. Am J Respir Cell Mol Biol 2006;35:175-81.

9. Andersson-Sjoland A, de Alba CG, Nihlberg K, et al. Fibrocytes are a potential source of lung fibroblasts in idiopathic pulmonary fibrosis. Int J Biochem Cell Biol 2008;40:2129-40.

10. Willis BC, Dubois RM, Borok Z. Epithelial origin of myofibroblasts during fibrosis in the lung. Proc Am Thorac Soc 2006; 3:377-82.

11. Willis BC, Liebler JM, Luby-Phelps K, et al. Induction of epithelial-mesenchymal transition in alveolar epithelial cells by transforming growth factor-beta1: potential role in idiopathic pulmonary fibrosis. Am J Pathol 2005;166:1321-32.

12. Kim KK, Kugler MC, Wolters PJ, et al. Alveolar epithelial cell mesenchymal transition develops in vivo during pulmonary fibrosis and is regulated by the extracellular matrix. Proc Natl Acad Sci 2006;103:13180-5.

13. Thiery JP, Sleeman JP. Complex networks orchestrate epithelial-mesenchymal transitions. Nat Rev Mol Cell Biol 2006;7:131-42.

14. Thiery JP. Epithelial-mesenchymal transitions in development and pathologies. Curr Opin Cell Biol 2003:15:740-6.

15. Nieto MA. The snail superfamily of zinc-finger transcription factors. Nat Rev Mol Cell Biol 2002;3:155-66.

16. Barrallo-Gimeno A, Nieto MA. The Snail genes as inducers of cell movement and survival: implications in development and cancer. Development 2005;132:3151-61.

17. Zavadil J, Bottinger EP. TGF-beta and epithelial-to-mesenchymal transitions. Oncogene 2005;24:5764-74.

18. Ando S, Otani $\mathrm{H}$, Yagi $Y$, et al. Proteinase-activated receptor 4 stimulation-induced epithelial-mesenchymal transition in alveolar epithelial cells. Respir Res 2007:8:31.

19. Kasai H, Allen JT, Mason RM, et al. TGF-beta1 induces human alveolar epithelial to mesenchymal cell transition (EMT). Respir Res 2005:6:56

20. Yao HW, Xie QM, Chen J0, et al. TGF-beta1 induces alveolar epithelial to mesenchymal transition in vitro. Life Sci 2004;76:29-37.
21. Xu GP, Li Q0, Cao XX, et al. The effect of TGF-beta1 and SMAD7 gene transfer on the phenotypic changes of rat alveolar epithelial cells. Cell Mol Biol Lett 2007;Epub ahead of print.

22. Kim JH, Jang YS, Eom KS, et al. Transforming growth factor beta1 induces epithelial-tomesenchymal transition of A549 cells. J Korean Med Sci 2007;22:898-904.

23. Königshoff $\mathbf{M}$, Kramer M, Balsara N, et al. WNT1-inducible signaling protein-1 mediates pulmonary fibrosis in mice and is upregulated in humans with idiopathic pulmonary fibrosis. J Clin Invest 2009;119:772-87.

24. Königshoff M, Balsara N, Pfaff EM, et al. Functional Wnt signaling is increased in idiopathic pulmonary fibrosis. PLOS ONE 2008:3:e2142.

25. Yu H, Königshoff M, Jayachandran A, et al. Transgelin is a direct target of TGF$\{$ beta\}/Smad3-dependent epithelial cell migration in lung fibrosis. FASEB J 2008;22:1778-89.

26. Thannickal VJ, Flaherty KR, Hyzy RC, et al. Emerging drugs for idiopathic pulmonary fibrosis. Expert Opin Emerg Drugs 2005:10:707-27.

27. Walter N, Collard HR, King TE Jr. Current perspectives on the treatment of idiopathic pulmonary fibrosis. Proc Am Thorac Soc 2006;3:330-8.

28. Phan SH. Biology of fibroblasts and myofibroblasts. Proc Am Thorac Soc 2008:5:334-7.

29. Selman M, King TE, Pardo A. Idiopathic pulmonary fibrosis: prevailing and evolving hypotheses about its pathogenesis and implications for therapy. Ann Intern Med 2001:134:136-51.

30. Scotton CJ, Chambers RC. Molecular targets in pulmonary fibrosis: the myofibroblast in focus. Chest 2007:132:1311-21.

31. Zeisberg $\mathbf{M}$, Kalluri R. The role of epithelial-to-mesenchymal transition in renal fibrosis. J Mol Med 2004;82:175-81.

32. Willis BC, Borok Z. TGF-beta-induced EMT: mechanisms and implications for fibrotic lung disease. Am J Physiol 2007;293:L525-34.

33. Peinado H, Quintanilla $\mathbf{M}$, Cano A. Transforming growth factor beta-1 induces snail transcription factor in epithelial cell lines: mechanisms for epithelial mesenchymal transitions. J Bio Chem 2003;278:21113-23.

34. Batlle $\mathbf{E}$, Sancho $\mathrm{E}$, Franci $\mathrm{C}$, et al. The transcription factor snail is a repressor of $\mathrm{E}$ cadherin gene expression in epithelial tumour cells. Nat Cell Biol 2000:2:84-9.

35. Shih JY, Tsai MF, Chang TH, et al. Transcription repressor slug promotes carcinoma invasion and predicts outcome of patients with lung adenocarcinoma. Clin Cancer Res 2005;11:8070-8.

36. Zhuo W, Wang $Y$, Zhuo $X$, et al. Knockdown of Snail, a novel zinc finger transcription factor, via RNA interference increases A549 cell sensitivity to cisplatin via JNK mitochondrial pathway. Lung Cancer 2008;62:8-14.

37. Lange-Sperandio B, Trautmann A, Eickelberg 0, et al. Leukocytes induce epithelial to mesenchymal transition after unilateral ureteral obstruction in neonatal mice. Am J Pathol 2007;171:861-71

38. Ward C, Forrest IA, Murphy DM, et al. Phenotype of airway epithelial cells suggests epithelial to mesenchymal cell transition in clinically stable lung transplant recipients. Thorax 2005;60:865-71.

39. Yamada M, Kuwano K, Maeyama T, et al. Dual-immunohistochemistry provides little evidence for epithelial-mesenchymal transition in pulmonary fibrosis. Histochem Cell Biol 2008;129:453-62.

\section{Quality \& Safety in Health Care}

Quality \& Safety in Health Care is a leading international peer-review journal in the growing area of quality and safety improvement. It provides essential information for those wanting to reduce harm and improve patient safety and the quality of care. The journal reports and reflects research, improvement initiatives and viewpoints and other discursive papers relevant to these crucial aims with contributions from researchers, clinical professionals and managers and experts in organisational development and behaviour.

qshc.bmj.com 